\title{
Enhanced photoluminescence excitation in surface plasmon coupling with an InGaN/GaN quantum well
}

\author{
Yen-Cheng Lu, Cheng-Yen Chen, Kun-Ching Shen, Dong-Ming Yeh, \\ Tsung-Yi Tang, and C. C. Yang ${ }^{\text {a) }}$ \\ Institute of Photonics and Optoelectronics and Department of Electrical Engineering, National Taiwan \\ University, 1, Roosevelt Road, Section 4, Taipei, Taiwan 10617, Republic of China
}

(Received 30 July 2007; accepted 14 October 2007; published online 31 October 2007)

\begin{abstract}
The authors report the observation of the enhancement of photoluminescence excitation through the couplings of an InGaN/GaN quantum well (QW) with localized surface plasmons (LSPs) and surface plasmon polaritons (SPPs), which are generated on a Ag nanostructure deposited on the SiN-coated QW epitaxial sample. At the wavelengths corresponding to the LSP modes, the excitation light is first absorbed by the LSPs. The LSP energy is then transferred into the QW such that the effective QW absorption is enhanced. Meanwhile, the application of the LSP local field to the QW may increase its absorption coefficient. Then, the coupling of the relaxed carriers with the SPPs enhances light emission that becomes stronger as temperature increases because of the increased carrier momentum. (C) 2007 American Institute of Physics. [DOI: 10.1063/1.2805034]
\end{abstract}

Recently, the enhancement of the spontaneous emission of an InGaN/GaN quantum well (QW) through the coupling of the dipole oscillators in the QW with surface plasmon polaritons (SPPs) on a nearby metallic structure has been demonstrated. ${ }^{1-7}$ Also, the enhancement of absorption efficiency of a semiconductor through the surface plasmon (SP) coupling near metallic nanoparticles has been reported. In particular, the excitation of SP on metal nanoparticles can enhance the absorption of nearby silicon. In this situation, the incident light first excites a localized surface plasmon on a metal nanoparticle. Then, the radiated localized surface plasmon (LSP) energy is absorbed by silicon to add to the direct photon absorption of silicon. Such an absorption enhancement has been used for improving the efficiency of a photodetector by $\mathrm{Ag}$ or $\mathrm{Au}$ nanoparticles in the device. ${ }^{8-10}$

In this letter, we report the observation of enhancing the excitation efficiency in the photoluminescence excitation (PLE) measurement through the SP-QW coupling process. The enhancement is due to the process of generating LSP modes through the absorption of the excitation light and hence effectively increasing QW absorption coefficient. ${ }^{11}$ Then, the carriers can couple with certain SPP modes for enhancing the emission, ${ }^{3,4}$ particularly when temperature is high. ${ }^{5}$ The used InGaN/GaN QW structure consists of a $3 \mathrm{~nm}$ well layer grown on a $2 \mu \mathrm{m}$ undoped GaN layer which is deposited on a $c$ plane sapphire substrate. A $10 \mathrm{~nm} \mathrm{GaN}$ cap layer is deposited on top of the QW. Such a sample is called the bare-QW sample. The other sample, called the $\mathrm{SiN} / \mathrm{Ag}$ coated sample, consists of a $10 \mathrm{~nm}$ SiN coating followed by a $50 \mathrm{~nm}$ Ag coating on the bare-QW sample.

Figure 1 shows the PL spectra of the bare-QW sample and the $\mathrm{SiN} / \mathrm{Ag}$-coated sample at 10 and $300 \mathrm{~K}$. One can see that with the $\mathrm{SiN} / \mathrm{Ag}$ coating, the PL intensity is enhanced at either 10 or $300 \mathrm{~K}$, particularly significant at $300 \mathrm{~K}$. Because the increase of PL excitation is expected to be weakly dependent on temperature, the significant difference in PL intensity enhancement between the cases of 10 and $300 \mathrm{~K}$ implies that the SP coupling plays an important role in the observed op-

\footnotetext{
${ }^{\text {a)} E l e c t r o n i c ~ m a i l: ~ c c y @ c c . e e . n t u . e d u . t w ~}$
}

tical behaviors. In particular, the SP coupling effect is stronger at $300 \mathrm{~K}$. In Fig. 1, one can also observe blueshifts in PL spectrum, particularly significant at $10 \mathrm{~K}$, upon the SiN/Ag coating. Here, it is interesting to see that the integrated PL intensity in the SiN/Ag coating sample at $300 \mathrm{~K}$ is higher than that in the bare-QW sample at $10 \mathrm{~K}$.

Figure 2 shows the normalized PLE intensities as functions of excitation wavelength at six labeled detection wavelengths of the two samples. The intensity shown in this figure represents the luminescence strength at the labeled wavelength when the sample is excited at the wavelength of the horizontal axis. At each detection wavelength, the PLE intensities of the two samples are normalized such that they have the same level at the first peak from the short excitation-wavelength end. With this normalization procedure, the PLE intensities of the SiN/Ag coated sample are generally higher than those of the bare-QW sample. In Fig. 2, as indicated with the short vertical lines, for each detection wavelength, the two samples have the peaks at the same excitation wavelength. The spectral locations of the six detection wavelengths can be connected with a straight line. The excitation wavelength spacing between two neighboring detection wavelengths is always around $5 \mathrm{~nm}$, which is con-

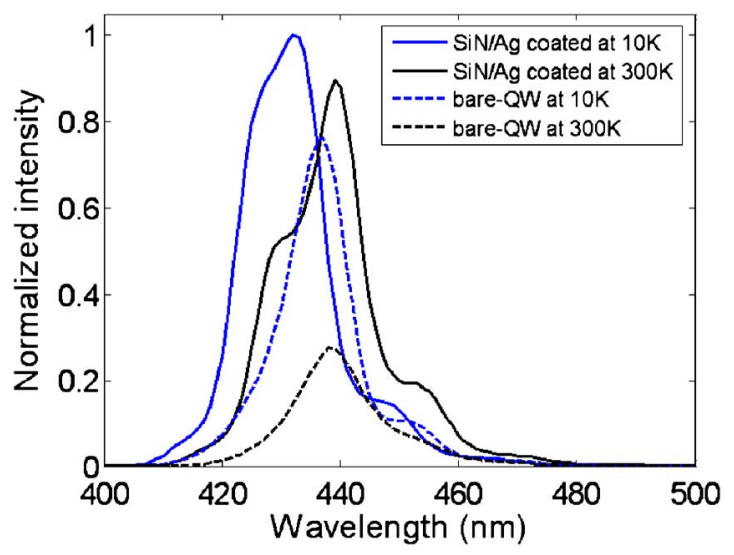

FIG. 1. (Color online) PL spectra of the bare-QW sample and the $\mathrm{SiN} / \mathrm{Ag}$-coated sample at 10 and $300 \mathrm{~K}$. 


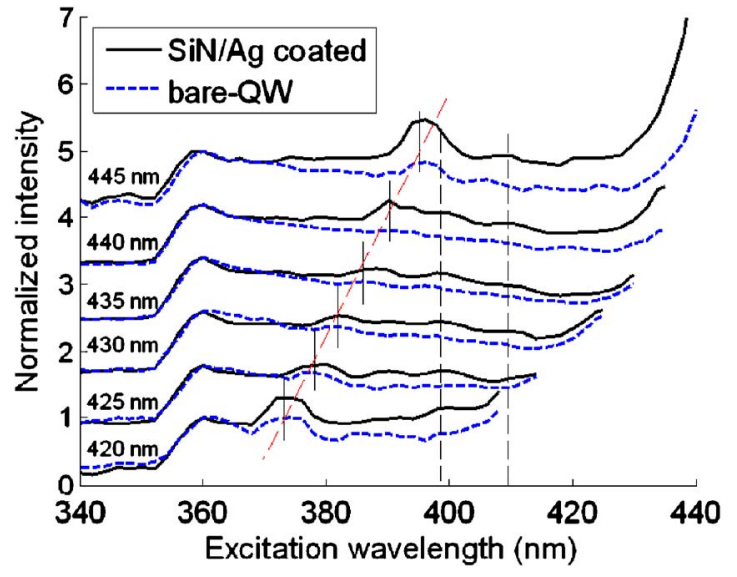

FIG. 2. (Color online) Normalized PLE signal intensities as functions of excitation wavelength at various detection wavelengths of the bare-QW sample and the SiN/Ag-coated sample at $10 \mathrm{~K}$.

sistent with the step size of the detection wavelength. This feature, which is common to the two samples, is attributed to the secondary diffraction contribution in the PLE measurement and has nothing to do with the SP-QW coupling process.

However, the two peaks, one at $398 \mathrm{~nm}$ and the other at $408 \mathrm{~nm}$, appear only in the curve of each detection wavelength of the SiN/Ag coated sample. These two peaks of fixed spectral positions in varying the detection wavelength are not seen in the bare-QW sample, implying that they are related to the SP-QW coupling. It is believed that the two peaks correspond to two LSP modes, which are generated on the Ag layer for coupling with the carriers in the QW to enhance the excitation in the PLE measurement. At these two excitation wavelengths, the excitation light is first absorbed by the LSP modes. Then, the energy of the LSP modes is transferred into the carriers in the QW through the absorptions of the LSP evanescent fields and the LSP-radiated photons. Therefore, besides the direct optical absorption of the QW, the SP-QW coupling creates another channel for carrier excitation in the QW at the energy levels higher than the PL emission states. Meanwhile, the application of the LSP local field to the semiconductor may directly increase its absorption coefficient. ${ }^{11}$

Normally, LSP modes exist on the metal structures of nanoparticle morphologies. ${ }^{8-11}$ The existence of the LSP modes with the resonance energies corresponding to 398 and $408 \mathrm{~nm}$ is supported by the atomic force microscopy (AFM) images on the surfaces of $\mathrm{SiN}$ and $\mathrm{SiN} / \mathrm{Ag}$ coatings, as shown in Figs. 3(b) and 3(c), respectively. For comparison, the AFM image on the surface of the bare-QW sample is also shown in Fig. 3(a). The roughness levels on the surfaces shown in Figs. 3(a)-3(c) are 0.17, 1.27, and $5.18 \mathrm{~nm}$, respectively. The nanostructures of a few tens of nanometers and those of around $100 \mathrm{~nm}$ in scale in Figs. 3(b) and 3(c), respectively, imply that $\mathrm{Ag}$ was coated in a morphology similar to nanoparticle. The existence of LSP in a nanograting structure has been numerically predicted, ${ }^{12,13}$ with the LSP resonance energies higher than that of SPP. Therefore, the LSP modes can exist in the SiN/Ag coated sample with a rough surface. Note that the SPP modes cannot exist in the energy range higher than its resonance energy at $\sim 2.9 \mathrm{eV}$ $(\sim 430 \mathrm{~nm})$. Meanwhile, the SPP modes cannot be excited directly through photon absorption without a prism or gratDownloaded 20 Feb 2009 to 140.112.113.225. Redistribution subject
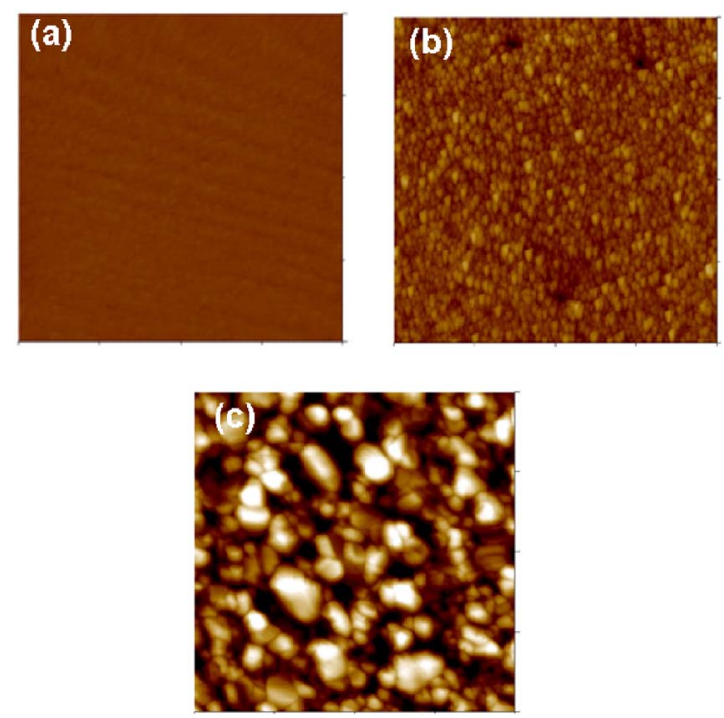

FIG. 3. (Color online) AFM images of the bare-QW sample (a), a SiNcoated sample (b), and the SiN/Ag-coated sample (c). The dimension of each image is $1 \times 1 \mu \mathrm{m}^{2}$.

ing scheme for momentum matching. On the other hand, a LSP mode, which does not have a significant momentum, can be excited through direct photon absorption. Therefore, it is believed that the LSP modes, instead of the SPP modes, play the major role in effectively enhancing photon absorption.

After the carriers in the QW are excited either by directly absorbing the excitation light or through the LSP-QW interaction, they will relax down to the lower levels for PL emission. Here, carriers can couple with SPP modes of lower resonance energies to enhance photon emission with the momentum matching between SPPs and photons through the rough metal surface. ${ }^{6}$ The coupling of the SPP modes with carriers relies on the availability of carrier momentum for momentum matching. Carrier momentum can be obtained from the thermal energy. Therefore, the energy transfer from carriers at the PL emission levels into the SPP modes and hence the enhancement of PL intensity are expected to increase with temperature. Figure 4 shows the temperature dependent normalized integrated PL intensities of the two samples. Here, one can see that the integrated PL intensity of the bare-QW sample drops to around $42 \%$ from 10 through $300 \mathrm{~K}$. On the other hand, the integrated PL intensity of the $\mathrm{SiN} / \mathrm{Ag}$ coated sample follows closely that of the bare-QW

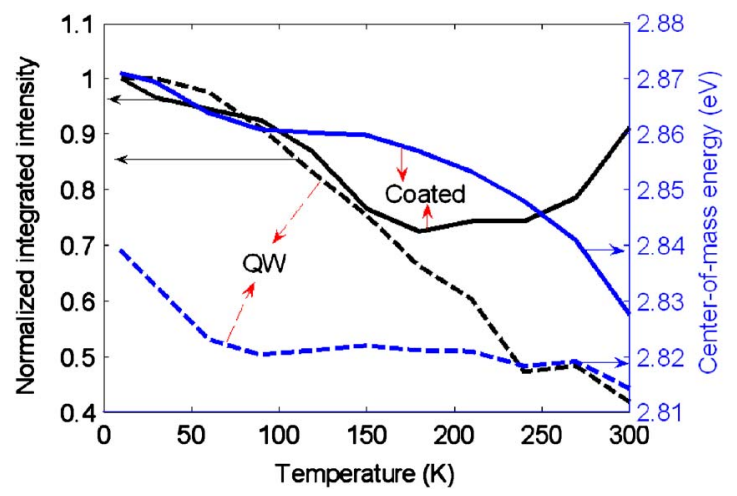

FIG. 4. (Color online) Temperature-dependent normalized PL integrated intensities and PL spectral center-of-mass energies of the bare-QW sample and the $\mathrm{SiN} / \mathrm{Ag}$ coated sample.

to AIP license or copyright; see http://apl.aip.org/apl/copyright.jsp 


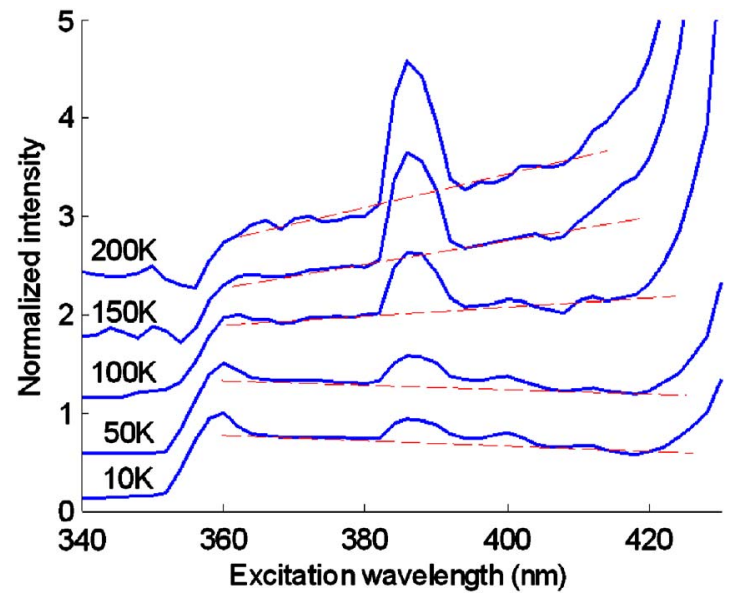

FIG. 5. (Color online) PLE intensities as functions of excitation wavelength at various temperatures of the $\mathrm{SiN} / \mathrm{Ag}$ coated sample.

sample up to $150 \mathrm{~K}$ and then turns around at $180 \mathrm{~K}$ to increase to about $90 \%$ at $300 \mathrm{~K}$. The turn around is due to the enhanced coupling between the carriers in the QW and the SPP modes as temperature increases. Because of the effective energy transfer from the relaxed carriers into the SPP modes and the efficient SP-photon coupling, the integrated PL intensity is increased up to the level of $90 \%$ the lowtemperature value.

In Fig. 4, we also show the temperature dependent PL spectral center-of-mass energies of the two samples. For the bare-QW sample, the typical $S$-shape variation of temperature-dependent PL spectral feature can be seen indicating the existence of composition fluctuation in the QW. ${ }^{14}$ For the SiN/Ag coated sample, the $S$-shape behavior can still be seen. However, even though the two center-of-mass energy curves are essentially parallel below $150 \mathrm{~K}$, beyond that, the PL spectral feature of the $\mathrm{SiN} / \mathrm{Ag}$ coated sample is rapidly redshifted as temperature increases. The redshift is attributed to the stronger coupling between the carriers and the SPP modes as temperature increases.

Figure 5 shows the PLE intensities as functions of excitation wavelength at various temperatures of the $\mathrm{SiN} / \mathrm{Ag}$ coated sample. The hump around $386 \mathrm{~nm}$ in each curve is an artifact in the PLE measurement, as already explained in
Fig. 2. The straight dashed lines show the increasing trend of the excitation wavelength dependence when temperature is higher than $50 \mathrm{~K}$. This trend is not observed in the bare-QW sample. The increasing trend in the $\mathrm{SiN} / \mathrm{Ag}$ coated sample confirms that the SP-QW coupling indeed becomes stronger as temperature increases.

In summary, we have observed the enhancement of PLE through the couplings of an InGaN/GaN QW with LSPs and SPPs on a Ag nanostructure deposited on the SiN-coated epitaxial sample. The effective QW absorption was enhanced through its absorptions of the LSP evanescent field and the LSP-radiated photons, and the coverage by LSP evanescence field for changing its absorption coefficient.

This research was supported by National Science Council, The Republic of China, under Grant Nos. NSC 95-2120M-002-012 and NSC 95-2221-E-002-287, and by U.S. Air Force Scientific Research Office under Contract No. AOARD-06-4052.

${ }^{1}$ K. Okamoto, I. Niki, A. Shvartser, Y. Narukawa, T. Mukai, and A. Scherer, Nat. Mater. 3, 601 (2004).

${ }^{2}$ K. Okamoto, I. Niki, A. Scherer, Y. Narukawa, T. Mukai, and Y. Kawakami, Appl. Phys. Lett. 87, 071102 (2005).

${ }^{3}$ C. Y. Chen, D. M. Yen, Y. C. Lu, and C. C. Yang, Appl. Phys. Lett. 89, 203113 (2006)

${ }^{4}$ C. Y. Chen, Y. C. Lu, D. M. Yeh, and C. C. Yang, Appl. Phys. Lett. 90, 183114 (2007).

${ }^{5}$ Y. C. Lu, C. Y. Chen, D. M. Yeh, C. F. Huang, T. Y. Tang, J. J. Huang, and C. C. Yang, Appl. Phys. Lett. 90, 193103 (2007).

${ }^{6}$ D. M. Yeh, C. Y. Chen, Y. C. Lu, C. F. Huang, and C. C. Yang, Nanotechnology 18, 265402 (2007).

${ }^{7}$ D. M. Yeh, C. F. Huang, Y. C. Lu, C. Y. Chen, T. Y. Tang, J. J. Huang, K. C. Shen, Y. J. Yang, and C. C. Yang, Appl. Phys. Lett. 91, 063121 (2007).

${ }^{8}$ K. R. Catchpole and S. Pillai, J. Lumin. 121, 315 (2006).

${ }^{9}$ S. Pillai, K. R. Catchpole, T. Trupke, and M. A. Green, J. Appl. Phys. 101, 093105 (2007).

${ }^{10}$ D. Derkacs, S. H. Lim, P. Matheu, W. Mar, and E. T. Yu, Appl. Phys. Lett. 89, 093103 (2006).

${ }^{11}$ D. M. Schaadt, B. Feng, and E. T. Yu, Appl. Phys. Lett. 86, 063106 (2005).

${ }^{12}$ W. C. Tan, T. W. Preist, and R. J. Sambles, Phys. Rev. B 62, 11134 (2000).

${ }^{13}$ W. C. Liu and D. P. Tsai, Phys. Rev. B 65, 155423 (2002).

${ }^{14}$ Y. S. Lin, K. J. Ma, C. Hsu, Y. Y. Chung, C. W. Liu, S. W. Feng, Y. C. Cheng, M. H. Mao, C. C. Yang, H. W. Chuang, C. T. Kuo, J. S. Tsang, and T. E. Weirich, Appl. Phys. Lett. 80, 2571 (2002). 